\title{
[Eu(1,2,4-BTC)], (1,2,4-BTC=1,2,4- benzen trikarboksilik asit) koordinasyon polimerinin kristal yapı ve optik özelliklerinin araştırılması
}

\author{
Adem DÖNMEZ ${ }^{1,2, *}$, Uğur ERKARSLAN ${ }^{1}$, Şehmus KARADENİZ ${ }^{1}$ \\ ${ }^{1}$ Muğla Sitkı Koçman Üniversitesi, Fen Fakültesi, Fizik Bölümü, Muğla. \\ ${ }^{2}$ Muğla Sitkı Koçman Üniversitesi, Bilimsel Araştırma Projeleri Koordinasyon Birimi, Muğla. \\ Geliş Tarihi (Recived Date): 18.08.2017 \\ Kabul Tarihi (Accepted Date): 13.11.2017
}

Özet

Metal kümelerden ve organik bağlayıcılarından oluşan gözenekli koordinasyon polimerleri foto-problar, organik ışı yayan diyotlar, sensörler, foto-katalizöterler ve diğer ışık yayımlayabilen aygıtlar gibi çeşitli geniş uygulama alanlartyla yeni sinı bir malzeme olarak malzeme bilimine umut vermektedir. Gözenekli koordinasyon polimerlerinin özelliklerinin, organik moleküller ile bağlantılı yapı taşlarını orantılı bir biçimde tasarlarken slkllkla değiştiği gözlemlenmiştir. Bu nedenle özel olarak sentezlenebilen yeni malzemeler ile yeni uygulama alanları keşfedilebilmekte ve bunların özellikleri geliştirilebilmektedir.

Bu çalı̧̧mada; yeni Eu(III) içeren gözenekli koordinasyon polimeri; [Eu(1,2,4-BTC)], (1,2,4-BTC=1,2,4Benzen trikarboksilik asit) hidrotermal yöntem ile sentezlenmiş ve toz kristal X-ışııı kırınımı, katı hal UV ve FT-IR spektroskopisi teknikleri ile yapısal olarak karakterize edilmiştir. Ayrıca oda sıcaklığında katı-hal fotolüminesans $(P L)$ özellikleri görünür bölge ve yakın klzıl ötesi bölgede ölçülmüss ve serbest liganddan elde edilen PL ölçümüyle karşslaş̧tırlmıştır. Sentezlenen malzeme NIR bölgede karakteristik şiddetli lüminesans özelliğe sahip olduğu için optik haberleşme, medikal teşhis ve diğer birçok teknolojik alanda potansiyel uygulama alanına sahip olabilir.

Anahtar Kelimeler: Lüminesans, gözenekli koordinasyon polimeri, Evropiyum.

\section{Investigation of crystal structure and optical properties of [Eu(1,2,4-BTC)], $(1,2,4-\mathrm{BTC}=1,2,4$-benzenetricarboxylic acid) coordination polymer}

\begin{abstract} synthesized for improving properties and discovering new applications.

\footnotetext{
* Adem DÖNMEZ, adonmez@mu.edu.tr, http://orcid.org/0000-0002-9773-0493

Uğur ERKARSLAN, egur@mu.edu.tr, http:///orcid.org/0000-0001-7667-2151

Sehmuz KARADENIZ, sehmuskaradenizz@gmail.com, http://orcid.org/0000-0002-7065-4507
}

Porous coordination polymers composed of metal clusters and organic binders have promised material science as a new class material with a wide range of applications such as photo-probes, organic light emitting diodes, sensors, photo-catalysis and other light emitting devices. It has been observed that the properties of porous coordination polymers frequently change when designing building blocks that are highly related to manipulative organic molecules. Therefore, the novel materials can be specifically

In this study, a new porous coordination polymer containing Eu-(III): [Eu(1,2,4-BTC)], (1,2,4-BTC=1,2,4Benzenetricarboxylic acid) were synthesized by hydrothermal method and characterized by powder X-ray diffraction, solid state UV and FT-IR spectra. In addition, the solid-state photoluminescence (PL) properties 
at room temperature were measured in the visible region and near infrared region and compared with the $P L$ measurement of its free ligand. Since the synthesized material has the characteristic strong luminescence properties in the NIR region, there could have many potential applications in optical communication, medical diagnosis and many other technological fields.

Keywords: Luminescence, porous coordination polymer, Europium.

\section{Giriş}

Yapıtaşları metal iyonları ve organik köprü ligandları olan, özgün bir uzaysal mimariye sahip makro moleküler malzemelere, "gözenekli koordinasyon polimerleri” adı verilir [1,2]. Bu tür malzemeler başlangıçta genel olarak gaz soğurma ve ayırmada uygulama alanı bulurken koordinasyon polimerlerinin tasarımının gelişmesiyle birlikte fonksiyonel hale getirilerek birçok alanda (hidrojen depolama, sensör, manyetik ve elektronik aygitlar ve biyomedikal gibi) kullanım imkanı bulmaktadırlar $[3,4]$. Lantanitlerden $\mathrm{Eu}^{+3}[\mathrm{Eu}(\mathrm{III})]$ iyonu, yoğun emisyon bantları, yüksek duyarlık ve uzun dalga boylu ışıma yaptığından dolayı f-f geçişlerinde uzun lüminesans ömrüne sahip olması, Eu bileşiklerini çeşitli optik uygulamalar için ilgi çekici hale getirir. Örneğin, bu bileşikler kırmızı 1 şı yayan elektrolüminesans cihazları, fotolüminesans işaretleme gibi alanlarda kullanılmaktadırlar [5]. Çeşitli organik ligandlarla Eu içeren malzemelerin elde edilmesi güçlü lüminesans özelliği gösteren yeni fonksiyonel malzeme üretimine olanak sağlayacağı için, bu malzemeler son zamanlarda yoğun şekilde çalışılmaktadır [6-11].

Bu çalışmada hidrotermal yöntem kullanılarak 1,2,4BTC ligandı ile Eu tabanlı malzeme olan $[\mathrm{Eu}(1,2,4-$ BTC)], (1,2,4-BTC=1,2,4- Benzentrikarboksilik asit) toz kristal halinde sentezlenmiş ve literatürde daha önceden rapor edilmiş benzer ligandlı tek kristal yapısı ile karşılaştırılmıştır [12]. Ayrıca, katı hal UV ve IR ölçümleri, görünür ve yakın kızılötesi bölgede ayrıntılı fotolüminesans analizleri yapılmış olup 1,2,4-BTC ligandının “Anten Etkisi” gösterdiği ve Eu(III) iyonun yaptığı lüminesansı şiddetlendirdiği ilk kez bu çalışmada sunulmuştur.

\section{Materyal ve metot}

Deneyde kullanılan kimyasal maddeler Merck, Fluka, ve Sigma-Aldrich firmalarından ticari olarak alınmıştır ve herhangi bir saflaştırma işlemi uygulamadan kullanılmıştır. Kompleksin ve ligandın; toz kristal $\mathrm{X}$-1şınları ölçümleri Philips X'Pert Pro marka toz kırınım cihazı ile $\mathrm{Cu}-\mathrm{K}_{\alpha}$ ışını kullanılarak oda sıcaklığında, IR spektrumları Perkin-Elmer Spectrum 65 FT-IR spektrometresi, katı UV ölçümleri Ocean Optics Maya 2000 Pro spektrometre ile yapılmıştır. Fotolüminesans ölçümleri ise Perkin Elmer LS 55 moleküler florometre ve Andor Solis SR 500i-BL model spektrometre ile oda sıcaklığında yapılmıştır. Emisyon spektrumları için uyarma kaynağı olarak Spectra Physics model Nd-YLF (349 nm) lazer kullanılmıştır. Her bir lazer atımı 5 ns atma genişliğinde ve $1,33 \mathrm{~mJ}$ enerjiye sahiptir.

\subsection{Eu(III) kompleksinin sentezlenmesi}

İlk olarak 1,2,4-Benzenetricarboxylic asit (1 mmol, 0,210 g) $10 \mathrm{ml}$ saf suda kaynama noktasına kadar 1sıtılarak çözülmüştür. Daha sonra bu çözeltiye $\mathrm{Eu}\left(\mathrm{Cl}_{3}\right)_{3} \times 5 \mathrm{H}_{2} \mathrm{O}(1 \mathrm{mmol}, 0,366)$ eklenip 20 dakika manyetik karıştırıcı ile karıştırılmıştır. Hazırlanan çözeltinin oda sıcaklığındaki ilk Ph değeri 2,60 olarak gözlenmiştir. Çözeltiye damla damla $\mathrm{NaOH}$ eklenerek Ph değeri 4,11 değerine yükseltilerek, çözelti tekrar manyetik karıştırıcıda kaynatılarak karıştırılmıştır. Elde edilen karışım yüksek basınca dayanıklı reaktöre koyulmuştur. Reaktör, oda sicaklığından $120{ }^{\circ} \mathrm{C}$ dereceye kadar 12 saatte 1sitılıp, 50 saat $120{ }^{\circ} \mathrm{C}$ sicaklıkta tutulup ve 24 saatte oda sicaklığına düşecek şekilde programlanan etüve yerleştirilmiştir. Üç gün sonra elde edilen toz kristaller süzme kâğıdıyla ayrıştırılmış. Eu(III) kompleksinin şematik gösterimi Şekil 2.1 de gösterilmektedir.

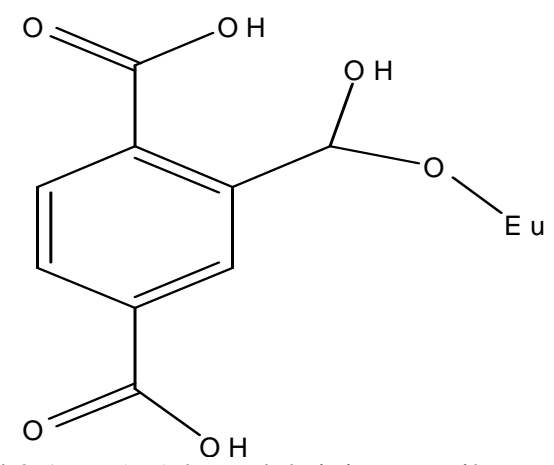

Şekil 2.1. Eu(III) kompleksinin şematik gösterimi.

\section{Araştırma bulguları}

\subsection{Eu(III) kompleksinin toz kristal ölçümü}

Eu(III) kompleksinin X-ışını toz kırınımı şiddet verilerinden elde edilen veriler, Mercury bilgisayar programı kullanılarak literatürdeki aynı ligand ile sentezlenmiş benzer tek kristal yapı ile karşılaştırılmıştır [12]. Toz kristal ölçümünden elde edilen grafik, "ölçülen" olarak, tek kristal verileri kullanılarak Mercury bilgisayar programı ile elde edilen grafik ise "hesaplanan" olarak isimlendirilmiş 
ve grafikler karşılaştırmalı olarak Şekil 3.1'de verilmiştir. Grafikten görüldüğü üzere; Eu(III) kompleksinde ölçülen ve hesaplanan piklerin uyumlu olduğu görülmüştür. Gözlenen piklerin uyumlu olması incelenen Eu(III) kompleksinin saf olduğunu göstermektedir.

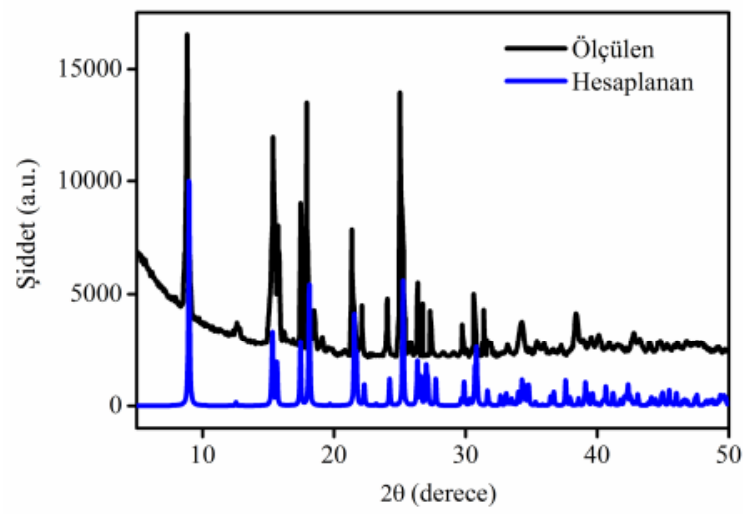

Şekil 3.1. Eu(III) kompleksi için X-ışını toz kırınımı desenleri

\subsection{Eu(III) kompleksinin UV ve IR analizi}

Eu(III) kompleksinin ve serbest ligandı 1,2,4-BTC nin katı halde UV spektrumları karşılaştırmalı olarak Şekil 3.2' de, IR spektrumları ise Şekil 3.3 de verilmiştir.

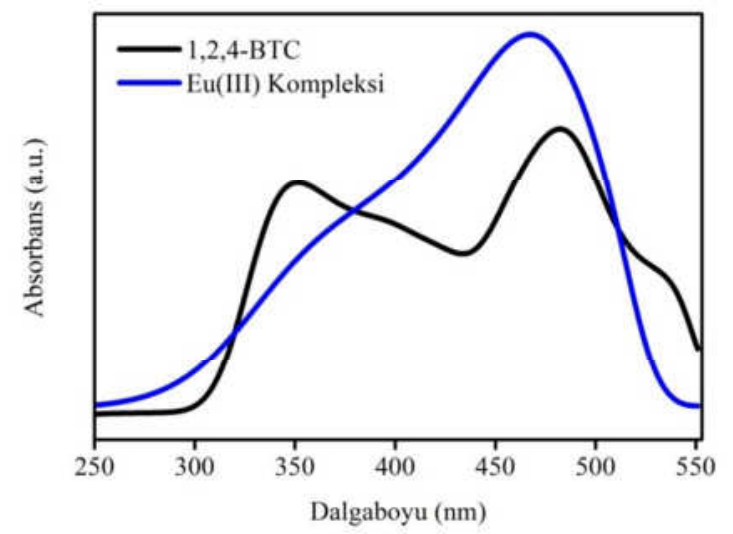

Şekil 3.2. 1,2,4-BTC ligandı ve Eu(III) kompleksinin UV spektrumları

Şekil 3.2 den görüldüğü gibi, 1,2,4-BTC ligandına ait $350 \mathrm{~nm}$ ve $482 \mathrm{~nm}$ maksimum dalgaboylarında iki adet bant gözlenirken, Eu(III) kompleksine ait $468 \mathrm{~nm}$ maksimum dalgaboyunda tek bant gözlenmiştir. Gözlenen soğurma bantları serbest ligandlardaki $\pi-\pi^{*}$ veya $\mathrm{n}-\pi^{*}$ geçişlerinden kaynaklanmaktadır [13].

Şekil 3.3 den görüldüğü üzere, 1,2,4-BTC ligandında gözlenmeyen fakat Eu(III) kompleksinin spektrumunda 3313-3095 $\mathrm{cm}^{-1}$ dalga boylarında gözlenen pikler koordine su moleküllerinin $v(\mathrm{O}-\mathrm{H})$ titreşimlerinden kaynaklanmaktadır [14]. 1,2,4-BTC ligandında görülen ancak $\mathrm{Eu}(\mathrm{III})$ kompleksinde gözlenmeyen $1687 \mathrm{~cm}^{-1}$ dalga boyundaki pik ise
$v(\mathrm{COOH})$ titreşiminden kaynaklanmaktadır ve bu grubun kompleksleşme ile protonlaştığını göstermektedir. Eu(III) kompleksinde $v_{\text {as }}(\mathrm{COO})$ ve $v_{\mathrm{s}}(\mathrm{COO})$ karboksilat gruplarının titreşimlerinden kaynaklanan pikler ise $1495-1409 \mathrm{~cm}^{-1}$ dalga boylarında oluşmaktadır. $\mathrm{Bu}$ titreşim pikleri arasındaki $86 \mathrm{~cm}^{-1}$ 'lik fark, karboksilat grubunun metal iyonu ile koordine olduğunu göstermektedir $[15,16]$.

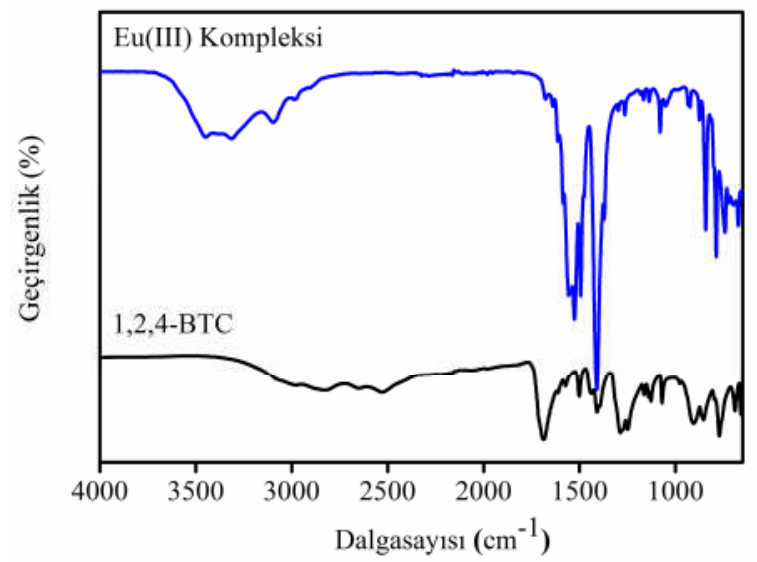

Şekil 3.3. 1,2,4-BTC ligandı ve Eu(III) kompleksinin IR spektrumları

3.3. Eu(III) kompleksinin ve 1,2,4-BTC ligandının katıhal fotolüminesans özellikleri

1,2,4-BTC serbest ligandı ve Eu(III) kompleksinin fotolüminesans ölçümleri oda sıcaklığında, katı halde ve $\lambda=349 \mathrm{~nm}$ lazer uyarması ile görünür ve NIR bölgede yapılmıştır. 1,2,4-BTC serbest ligandının emisyon spektrumu Şekil 3.4 de, Eu(III) kompleksinin görünür bölge emisyon spektrumu Şekil 3.5 de ve NIR bölge emisyon spektrumu ise Şekil 3.6 da verilmektedir.

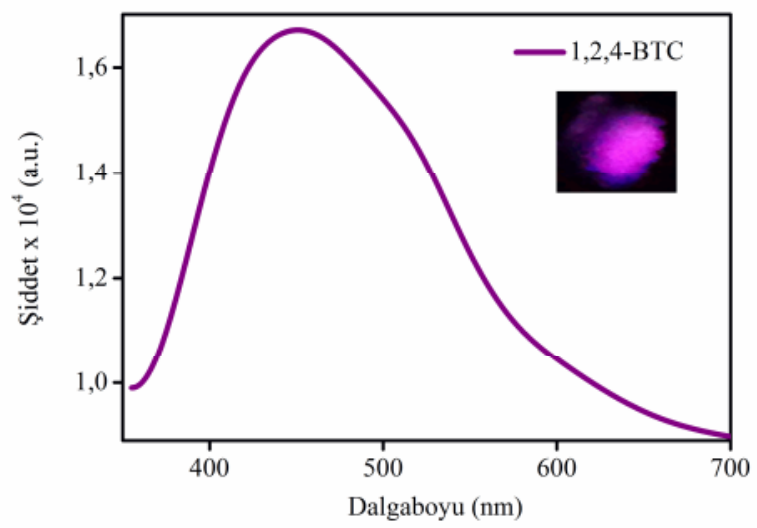

Şekil 3.4. 1,2,4-BTC ligandına ait emisyon spektrumu. Sağ üstteki fotoğraf; 1,2,4-BTC ligandının $349 \mathrm{~nm}$ lazer ile uyarılırken yaydı ğı fotolüminesans 1şımanın görüntüsüdür.

Şekil 3.4 den görüldüğü gibi, 1,2,4-BTC ligandı şiddetli mor 1şıma yapmaktadır. Geniş bant spektrumunun maksimum olduğu dalga boyu 450 
$\mathrm{nm}$ dir. $\mathrm{Bu}$ maksimum dalga boyuna karşılık gelen geçişler $n \rightarrow \pi^{*}$ veya $\pi \rightarrow \pi *$ elektronik geçişlerinden kaynaklanmaktadır [13].

Şekil 3.5 den görüldüğü gibi, Eu(III) kompleksine ait görünür bölgede beş karakteristik ${ }^{5} \mathrm{D}_{0} \rightarrow{ }^{7} \mathrm{~F}_{\mathrm{J}}$ keskin-bant emisyon piki vardır. $616 \mathrm{~nm}, 593 \mathrm{~nm}$, $652 \mathrm{~nm}$ ve $698 \mathrm{~nm}$ dalga boylarındaki ışımalar sirasiyla, ${ }^{5} \mathrm{D}_{0} \rightarrow{ }^{7} \mathrm{~F}_{2}{ }^{5} \mathrm{D}_{0} \rightarrow{ }^{7} \mathrm{~F}_{1}{ }^{5} \mathrm{D}_{0} \rightarrow{ }^{7} \mathrm{~F}_{3}$ ve ${ }^{5} \mathrm{D}_{0} \rightarrow{ }^{7} \mathrm{~F}_{4}$ geçişlerine karşılık gelmektedir. $\mathrm{Eu}(\mathrm{III})$ kompleksinin NIR bölgedeki emisyon spektrumunda ise $1160 \mathrm{~nm}, 1210 \mathrm{~nm}$ ve $1370 \mathrm{~nm}$ dalga boylarındaki keskin pikler sırasıyla ${ }^{7} \mathrm{~F}_{6} \rightarrow{ }^{7} \mathrm{~F}_{0}$, ${ }^{7} \mathrm{~F}_{5} \rightarrow{ }^{7} \mathrm{~F}_{0}$ ve ${ }^{7} \mathrm{~F}_{4} \rightarrow{ }^{7} \mathrm{~F}_{0}$ geçişleri sonucunda ortaya çıkmaktadır (Şekil 3.6).

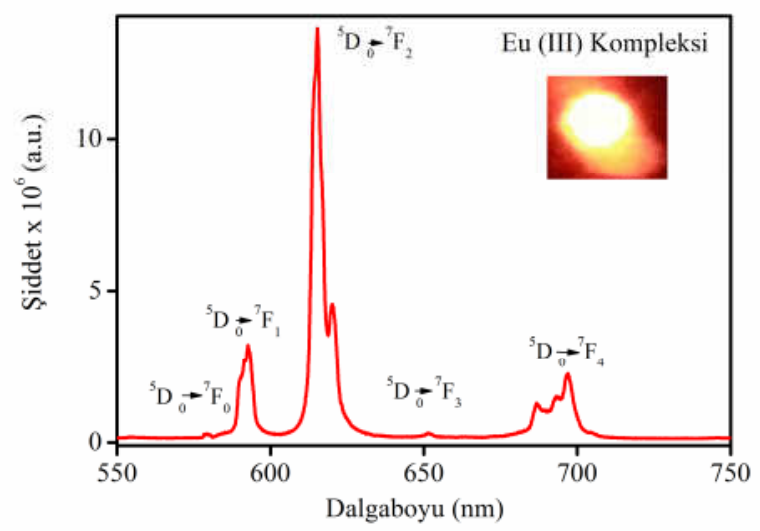

Şekil 3.5. Eu(III) kompleksinin görünür bölge emisyon spektrumu. Sağ üstteki fotoğraf; Eu(III) kompleksinin $349 \mathrm{~nm}$ lazer ile uyarılırken yaydığ fotolüminesans 1şımanın görüntüsüdür.

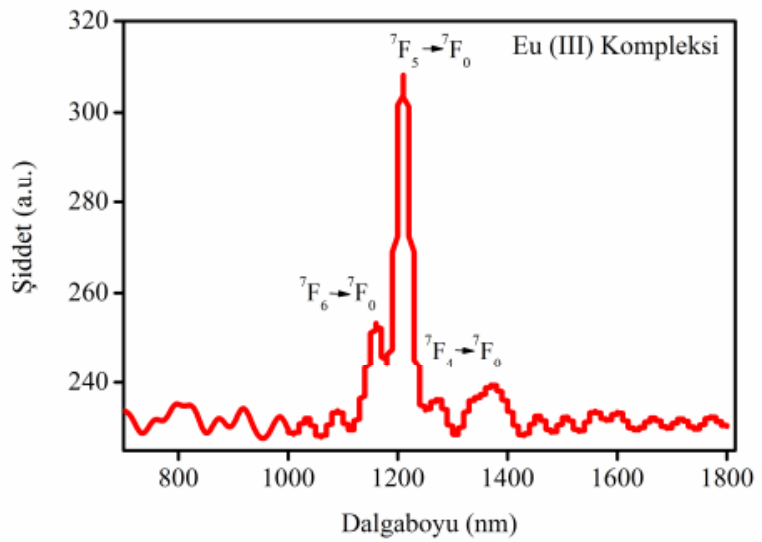

Şekil 3.6. Eu(III) kompleksinin NIR bölge emisyon spektrumu

\subsection{Eu(III) kompleksi için enerji diyagramı ve "Anten" etkisi}

Genel olarak ligandlar için geniş bant spektrumu, lantanitler için ise daha keskin spektrumlar gözlenir. Lantanitlerin f-f yasaklı geçişleri nedeniyle düşük soğurma kapasiteleri vardır ve ligandlar ile kompleks oluşturduklarında yüksek soğurma yapabilen ve lantanitin keskin piklerine sahip malzemeler elde edilir. Eu(III) kompleksinin görünür bölge ve NIR spektrumları incelendiğinde ligand kaynaklı herhangi bir geniş bant emisyon tespit edilememesi 1,2,4-BTC ligandı tarafından soğrulan enerjinin Eu(III) iyonuna verimli şekilde aktarıldığı anlamına gelir.

Konjuge ligandlar içeren Eu(III) komplekslerinin emisyon durumları genellikle intramoleküler enerji transfer prosesi yoluyla veya ligandların triplet uyarılmış durumları ile ortaya çıkar (Şekil 3.7). $\mathrm{Eu}(\mathrm{III})$ kompleksi için şiddetli lüminesans elde edilmesi 1şınımsız bozunum ile enerji kaybını azaltan ve ligandın rijitliğini verimli şekilde arttıran, metal merkez ile ligandın bağlanması veya kompleksleşmesi ile ilişkilendirilebilir.

Dexter teorisine göre; ligandın uyarılmış enerji seviyeleri ile $\mathrm{Ln}^{+3}$ iyonunun triplet durumu arasındaki enerji farkı, etkin enerji aktarımında anahtar rolü oynamaktadır $[17,18]$. Bu iki seviye arasındaki enerji farkı çok büyük olursa ligand ve $\mathrm{Ln}^{+3}$ iyonunun triplet seviyelerinin üst üste çakıșması azalacak ve enerji aktarımı keskin bir şekilde düşecektir. Triplet seviyeler arasındaki fark çok küçük olursa, bu durumda da lantanit iyonundan liganda enerjinin geri transfer süreci başlayacaktır. 1,2,4-BTC ligandının triplet enerji seviyeleri, Eu(III) iyonunun $4 \mathrm{f}$ seviyelerine enerji aktarımı için karşılaştırılabilir değerlerdedir (Şekil 3.7). Sonuç olarak liganddan da Eu(III) e etkin olarak enerji aktarımı gerçekleşmiştir.
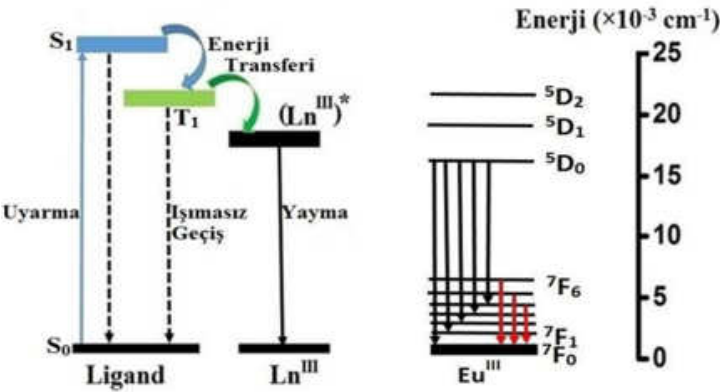

Şekil 3.7. Eu(III) kompleksine ait enerji diyagramı [19].

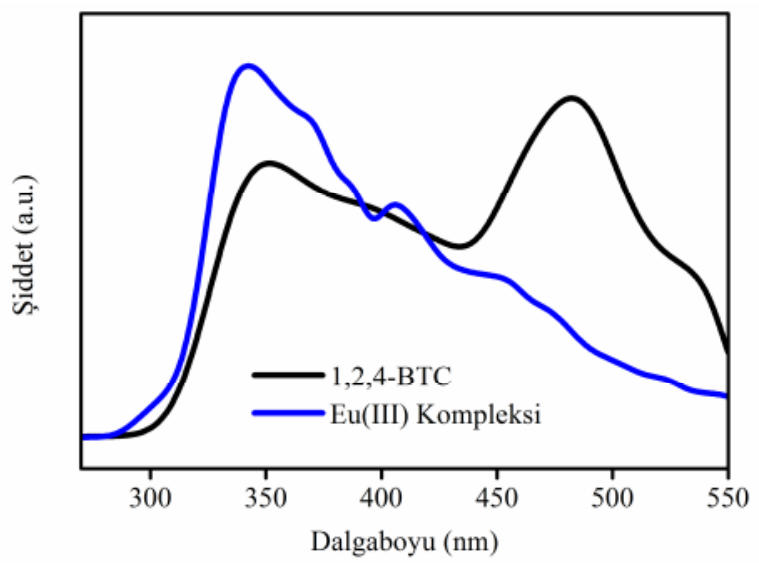

Şekil 3.8: Eu(III) kompleksinin uyarılma ve 1,2,4BTC ligandının soğurma spektrumları 
Şekil 3.8' de Eu(III) kompleksinin uyarılma ve 1,2,4-BTC ligandının soğurma spektrumları gösterilmiştir. Spektrumların iyi şekilde örtüşmesi, liganddan $\mathrm{Eu}(\mathrm{III})$ iyonuna enerji transferinin gerçekleştiğini ve 1,2,4-BTC ligandının "Anten etkisi" ni göstermektedir.

\section{Tartışma ve sonuç}

Eu(III) tabanlı gözenekli koordinasyon polimeri; [Eu(1,2,4-BTC)], 1,2,4-BTC=1,2,4- Benzen trikarboksilik asit ligandı kullanılarak hidrotermal yöntem ile sentezlenmiştir. Toz kristal X-1şını kırınımı, katı hal UV ve FT-IR spektroskopisi teknikleri ile yapısal olarak karakterize edilmiştir. $\mathrm{Bu}$ çalışmanın temel amacı doğrultusunda katı hal lüminesans özellikleri, 1,2,4-BTC ligandı ve Eu(III) kompleksi için oda sicaklığında, görünür ve NIR bölgede incelenmiştir. Fotolüminesans ölçümleri

\section{Kaynaklar}

[1] Natarajan, S. ve Mahata, P., Metal-organic framework structures - how closely are they related to classical inorganic structures, Chemical Society Reviews, 38, 2304-2318, (2009).

[2] Qiu, S.L. ve Zhu, G.S., Molecular engineering for synthesizing novel structures of metal-organic frameworks with multifunctional properties, Coordination Chemistry Reviews, 253, 2891-2911, (2009).

[3] Yabing H., Wei Z., Guodong Q. ve Banglin C., Methane storage in metal-organic frameworks, Chemical Society Reviews, 43, 5657, (2014).

[4] Lu, G. ve Hupp, J.T., Metal-Organic frameworks as sensors: A ZIF-8 based fabryperot device as a selective sensor for chemical vapors and gases, Journal of Amarican Chemical Society, 132, 78327833, (2010).

[5] Karadeniz, Ş., Evropiyum içeren lüminesans özelliği gösteren metal-organik malzemelerin üretimi ve yapılarının araştırılması. Yüksek Lisans Tezi, Muğla Sıtkı Koçman Üniversitesi, Fen Bilimleri Enstitüsü, Muğla, (2017).

[6] De Sa', G.F., Malta, O.L., de Mello D.C., Simas, A.M., Longo, R.L., Santa-Cruz P.A. ve de Silv, E.F., Spectroscopic properties and design of highly luminescent lanthanide coordination complexes, Coordination Chemistry Reviews, 196, 165-195, (2000).

[7] Wang, Z., Streobele, M., Zhang, K.L., Meyer H.J., You, X.Z. ve Yu, Z., A new family of two-dimensional lanthanide(III) coordination sonucunda, Eu(III) kompleksi şiddetli kırmızı 1şıma verirken, 1,2,4-BTC ligandı ise mor 1şıma vermiştir. Yapılan analizler sonucunda 1,2,4-BTC ligandının "Anten Etkisi" gösterdiği ve Eu(III) iyonun yaptığı lüminesansı şiddetlendirdiği gözlenmiştir ve bu olay enerji transfer mekanizması ile ayrıntılı olarak açıklanmıştır. Sentezlenen Eu(III) tabanlı malzeme, NIR bölgede karakteristik şiddetli lüminesans özelliğe sahip olduğu için optik haberleşme, medikal teşhis ve diğer birçok teknolojik alanda potansiyel uygulama alanına sahip olabilir [20-23].

\section{Teşekkür}

Yazarlar, Muğla Sıtkı Koçman Üniversitesi Bilimsel Araştırma Projeleri Birimi tarafından 16/042 nolu proje ile desteklendiği için kendilerine, ayrıca fotolüminesans ölçümleri için Balıkesir Üniversitesi Bilim ve Teknoloji Uygulama ve Araştırma Merkezine ve Dr. Mustafa Burak ÇOBAN'a teșekkür eder.

polymers: synthesis, structures and properties of $[\mathrm{Ln}(\mathrm{SIP})(\mathrm{H} 2 \mathrm{O}) 4] \mathrm{n}(\mathrm{Ln}=\mathrm{Eu}, \mathrm{Gd}, \mathrm{Ce}$, and $\mathrm{NaH} 2 \mathrm{SIP}=5$-sulfoisophthalic acid monosodium salt), Inorganic Chemistry Communications, 5, 230-234, (2002).

[8] Hua-Bin, Z., Chong-Bin, T., Shu-Ting, W., Jian-Di L., Zhi-Hua, L. ve Shao-Wu, D., Synthesis, structures and physical properties of new 3D lanthanide coordination polymers constructed from 1,2,4,5benzenetetracarboxylic acid, Journal of Molecular Structure, 985, 355-360, (2011).

[9] Li-Juan, H., Ya-Jie, K., Ning, S. ve XingLing, J., A new europium fluorous metalorganic framework with pentafluorobenzoate and 1,10-phenanthroline ligands: Synthesis, structure and luminescent properties, Journal of Flourino Chemistry, 166, 122-126, (2014).

[10] Hui, B., Zhenting, W. ve Ming, H., Lanthanide metal-organic frameworks with 2,2-bipyridinepolycarboxylic acid: Synthesis, crystal structures and fluorescent properties, Inorganica Chimica Acta, 427, 112-117, (2015).

[11] Yi-Xia, R., Miao, A., Hong-Mei, C., Mei-Li, Z. ve Ji-Jiang, W., Cooperation of coordinative and $\pi \cdots \pi$ interaction in directing two-fold interpenetrated architecture of two Eu/Dy(III) 2-sulfoterephthalate complexes with 4,4-bipyridine, Zeitschrift für Anorganische und Allgemeine Chemie, 641, 525-528, (2015).

[12] Surblé, S., Serre, C., Millange, F. ve Férey, G., Structural changes upon dehydration of $\mathrm{Pr}(\mathrm{III})(\mathrm{H} 2 \mathrm{O})\{\mathrm{C} 6 \mathrm{H} 3-(\mathrm{CO} 2) 3\}$ or MIL-81: A new three-dimensional praseodymium 1,2,4benzenetricarboxylate with a one dimensional 
inorganic sub-network, Solid State Sciences, 8, 413-417, (2006).

[13] Feng, X., Feng, Y.Q., Chen, J., Ng, S.W., Wang, L.Y. ve Guo, J.Z., Reticular threedimensional $3 \mathrm{~d}-4 \mathrm{f}$ frameworks constructed through substituted imidazole-dicarboxylate: syntheses, luminescence and magnetic properties study, Dalton Transactions, 44, 804-816, (2015).

[14] Lyszczek, R.M., Thermal investigations of cefadroxil complexes with transition metals, Journal of Thermal Analysis and Calorimetry, 78, 473, (2004).

[15] Li, X., Lu, Y., Bing, Y. ve Zha, M-Q., Synthesis, photoluminescent, and magnetic properties of two lanthanide sulfosalicylate complexes, Synthesis and Reactivity in Inorganic, Metal-Organic and Nano-Metal Chemistry, 42, 698-704, (2012).

[16] Li, X., Xie, Z., Lin, J. ve Cao, R., Lanthanide-organic frameworks constructed from multi-functional ligands: Syntheses, structures, near-infrared and visible photoluminescence properties, Journal of Solid State Chemistry, 182, 2290-2297, (2009).

[17] Dexter, D.L., A theory of sensitized luminescence in solids, Journal of Chemical Physics, 21, 836-850, (1953).

[18] Dang, S., Sun, L., Zhang, H., Guo, X-M., Li, Z., ve Feng, J., Near-1nfrared luminescence from sol-gel materials doped with Holmium(III) and Thulium(III) complexes,
Journal of Physical Chemistry C, 112, 13240-13247, (2008).

[19] Çoban M.B., Erkarslan U., Oylumluoglu G., Aygün $M$ ve Kara H., Hydrothermal synthesis, crystal structure and photoluminescent properties; 3D Holmium(III) coordination polymer, Inorganica Chimica Acta, 447, 87-91, (2016)

[20] Monteiro, J.H.S.K., Machado, D., Hollanda, L.M., Lancellotti M., Sigoli F.A. and Bettencourt-Dias A. de, Selective cytotoxicity and luminescence imaging of cancer cells with a dipicolinato-based EuIII complex, Chemical Communications, 53, 86, 11818-11821, (2017).

[21] Kanazawa, K., Komiya, Y., Nakamura, K. and Kobayashi, N., Red luminescence control of Eu(III) complexes by utilizing the multicolored electrochromism of viologen derivatives, Physical Chemistry Chemical Physics, 19, 26, 16979-16988, (2017).

[22] Abdollahi, S., Harris, W.R. and Riehl, J.P., Application of circularly polarized luminescence spectroscopy to $\mathrm{Tb}$ (III) and $\mathrm{Eu}(\mathrm{III})$ complexes of transferrins, Journal of Physical Chemistry, 100, 5, 1950-1956, (1996).

[23] Sheng-Gui, L., Qing, W., Guo-Xian, S. and Xiao-Bei, D., Luminescence and application in light-emitting diodes of dinuclear Eu(III) complex based on 4,4'-bis(4",4",4"-trifluoro1",3"-dioxobutyl)-o-terphenyl, Acta Chimica Sinica, 68, 1, 62-66, (2010). 